\title{
Medical Image Segmentation Using Multi-level Set Partitioning with Topological Graph Prior
}

\author{
Saif Dawood Salman Al-Shaikhli, Michael Ying Yang, and Bodo Rosenhahn \\ Appelstr. 9A, 30167 Hannover, Germany \\ \{shaikhli, yang, rosenhahn\}@tnt.uni-hannover.de \\ http://www.tnt.uni-hannover.de
}

\begin{abstract}
In this paper, we propose an approach for multi-region segmentation based on a topological graph prior within a multi-level set (MLS) formulation. We consider topological graph prior information to evolve the contour based on a topological relationship presented via a graph relation. This novel method is capable of segmenting adjacent objects with very close gray level that would be difficult to segment correctly using standard methods. We describe our algorithm and show the graph prior technique to explain how it gives precise multi-region segmentation. We validate our algorithm with numerous abdominal and brain image databases and compare it to other multi-region segmentation methods to demonstrate its accuracy and computational efficiency.
\end{abstract}

Keywords: Segmentation, multi-region, topological graph, level set, medical image.

\section{Introduction}

Medical imaging of internal organs of the human body is important to improve medical diagnosis and therapy. Multi-region image segmentation is a major task in medical imaging. Due to poor resolution and weak contrast this task is difficult in the presence of noise and artifacts [1]. Many existing methods for segmentation are based on image intensity information, shape properties or shape priors [1], [2], 3], 4].

M. Suzuki et al. [5] propose abdominal multi-organ segmentation with analyses of missing organs using statistical location model. A. Shimizua et al. 6] propose simultaneous extraction of multiple organs from abdominal CT using abdominal cavity standardization process with feature database and atlas guided segmentation incorporating parameter estimation for organ segmentation. M. G. Linguraru et al. 7] propose multi-region segmentation using graph cut method for four abdominal organ segmentation. T. Kohlberger et al. [8] propose multiorgan segmentation from CT medical images using learning-based segmentation and shape representation. Okada et al. 9] propose multi-organ segmentation based on hierarchical spatial modeling of organ interrelations using atlas information. P. L. Bazin et al. 10] propose multi-region segmentation algorithm of 
brain image using topological and statistical atlases of brain as prior to the segmentation framework. Compared to the existing works, our proposed topological prior is much more high level but still gives accurate results with less computational time and does not need training data required for statistical methods.

The contribution of our approach is multi-region segmentation using a multilevel set method with graph prior by representing the objects relation in the image as topological graph: therefore, we determine the location and the area of each region as well as the topological correlation and discrimination between different regions in the image. The graph prior is embedded in the multi-level set energy equation and acts as an additional prior term to identify both the overlapped regions and weak boundaries between adjacent regions in the image as shown in Fig. 11 The graph priors allow us to handle the huge variability of medical image data in more abstract fashion. Consequently, our algorithm is less sensitive to noise and gives accurate segmentation of ambiguous regions depending on the topological correlation of different regions in the image.

The rest of the paper is organized is as follows: Section 2 contains the explanation of the graph representation and how this prior integrated in the energy functional. In Section 3 we discuss the experimental results. Finally we conclude and summarize our work in Section 4.

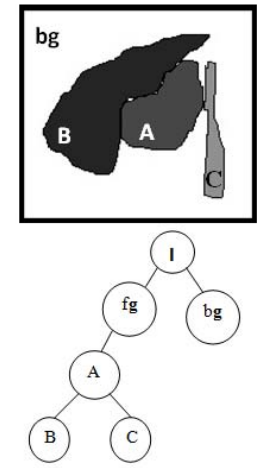

(a) Input image and its topological graph

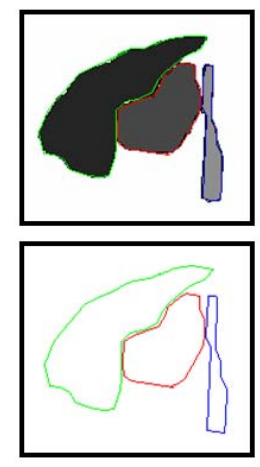

(b) With graph prior
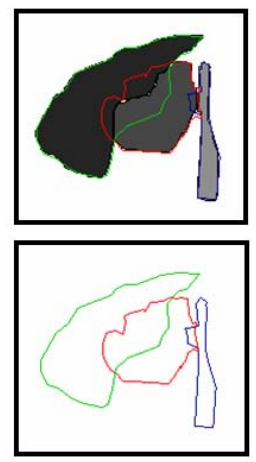

(c) Without graph prior

Fig. 1. Toy example of three region segmentation with and without topological graph prior

\section{Method}

In this section we will explain our proposed method for multi-region segmentation based on a multi-level set formulation with a topological graph prior.

\subsection{Graph Prior}

Human body organs have specific topological correlations between them and according to these correlations the exact location and boundary of these organs 
can be determined. If we consider the image $B$ as sets of clusters (segments) $B=\zeta_{i}, \zeta_{i+1}, \ldots, \zeta_{N}$ depending on the dissimilarity between them and $\chi_{\zeta_{i}}$ is the membership function of each cluster. These clusters are connected with each other by a specific topological relationship then the topological graph of these clusters can give information like the area, the location and the topological relationship of each cluster in the image. The topological graph is constructed from the test image firstly to provide the prior knowledge to the system. Figure 2 explains the representation of the anatomical structures in the image as topological graph. The clusters in the topological graph of the image $B$ are determined using Otsu's method [11] and these clusters are labeled according to their topological relationship. Let $\zeta^{\circ}$ be the interior of the cluster, $\zeta^{c}$ be the exterior of the cluster and $\partial \zeta$ be the boundary of the cluster. The topological relationship between the clusters is calculated in terms of probability of intersections of these clusters [12, [16] as follows:

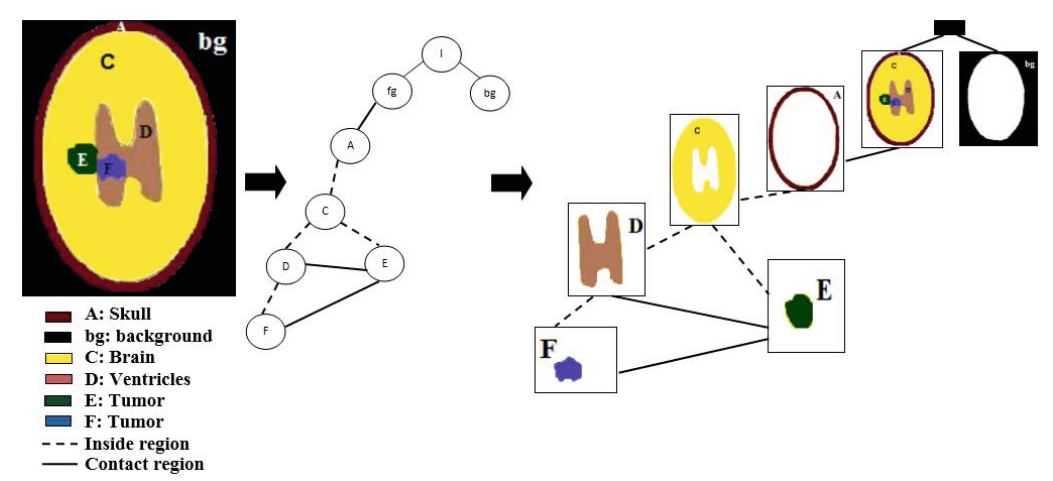

Fig. 2. Image representation as topological graph

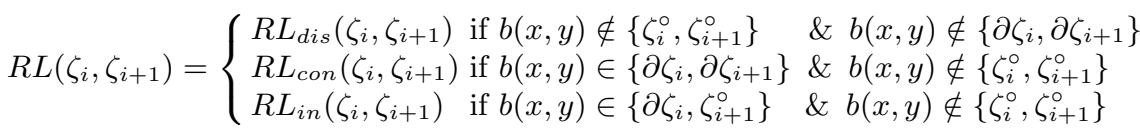

$$
\begin{aligned}
& R L_{d i s}\left(\zeta_{i}, \zeta_{i+1}\right)=1-\max _{b}\left\{\left|\chi_{\zeta_{i}}(b(x, y))+\chi_{\zeta_{i+1}}(b(x, y))-1\right|\right\} \\
& R L_{c o n}\left(\zeta_{i}, \zeta_{i+1}\right)=\min \left\{\left(1-\max _{b}\left(\left|\chi_{\zeta_{i}^{\circ}}(b(x, y))+\chi_{\zeta_{i+1}^{\circ}}(b(x, y))-1\right|\right)\right),\right. \\
& \left.\max _{b}\left(\min \left(\chi_{\partial \zeta_{i}}(b(x, y)), \chi_{\partial \zeta_{i+1}}(b(x, y))\right)\right)\right\} \\
& R L_{i n}\left(\zeta_{i}, \zeta_{i+1}\right)=\min \left(1, \min _{b}\left(1+\chi_{\zeta_{i+1}^{\circ}}(b(x, y))-\chi_{\zeta_{i}}(b(x, y))\right)\right)
\end{aligned}
$$

where $R L$ is region relationship, $R L_{d i s}, R L_{c o n}$ and $R L_{i n}$ are disjoint, contact and inside region relationship respectively. $b(x, y)$ are pixels in the image $B . x$ 
and $y$ represent the location of the pixel $b$ in the image $B$. The topological region relationship $T R L$ of the clusters are represented by 9 -intersection model in $3 \times 3$ matrix:

$$
\operatorname{TRL}\left(\zeta_{i}, \zeta_{i+1}\right)=\left(\begin{array}{ccc}
a_{11}\left(\zeta_{i}^{\circ} \bigcap \zeta_{i+1}^{\circ}\right) & a_{12}\left(\zeta_{i}^{\circ} \bigcap \partial \zeta_{i+1}\right) & a_{13}\left(\zeta_{i}^{\circ} \bigcap \zeta_{i+1}^{c}\right) \\
a_{21}\left(\partial \zeta_{i} \bigcap \zeta_{i+1}^{\circ}\right) & a_{22}\left(\partial \zeta_{i} \bigcap \partial \zeta_{i+1}\right) & a_{23}\left(\partial \zeta_{i} \bigcap \zeta_{i+1}^{c}\right) \\
a_{31}\left(\zeta_{i}^{c} \bigcap \zeta_{i+1}^{\circ}\right) & a_{32}\left(\zeta_{i}^{c} \bigcap \partial \zeta_{i+1}\right) & a_{33}\left(\zeta_{i}^{c} \bigcap \zeta_{i+1}^{c}\right)
\end{array}\right)
$$

Each element in Eq.5represents specific topological relationship. For example, if $a_{11}=0, a_{12}=0, a_{21}=0$ and $a_{22}=0$, which means that all pixels in cluster $\zeta_{i}$ are not in $\zeta_{i+1}$, then these regions must be disjoint regions. If $a_{21}=1$, which means that the pixels in $\partial \zeta_{i}$ are in $\zeta_{i+1}$, then $\zeta_{i}$ is inside $\zeta_{i+1}$. If $a_{11}=0$ and $a_{22}=1$, which mean that the pixels in $\partial \zeta_{i}$ are in $\partial \zeta_{i+1}$, then these two regions are contact in their boundaries. If and only if $a_{12}=1$, then this region has an internal cavity and it handles another region. Table 1 summarizes how each element of the matrix in Eq. 5] determines the relationship between the clusters by checking the primary conditions and the secondary conditions. The primary conditions are the main conditions to determine the topological relationship between the regions. Table 2 shows $T R L$ of each region in the image of Fig. 2 . The connected components represent the total relationship of each region. The number of cavities in each region indicates how many regions are inside it or how many regions are held by it. For example, in case of region $E$ in Fig. 2, it has three relationships (three connected components) $T R L_{1}(E, F), T R L_{2}(E, D)$ and $T R L_{3}(E, C)$ as in Eq. 6. $T R L_{1}(E, F)$ and $T R L_{2}(E, D)$ show that $E$ is in contact with $D$ and $F$ while $T R L_{3}(E, C)$ shows that $E$ is inside $C$ according to Eq. 5.

$$
\begin{gathered}
T R L_{1}(E, F)=\left(\begin{array}{lll}
0 & 0 & 0 \\
0 & 1 & 1 \\
0 & 1 & 1
\end{array}\right), \quad T R L_{2}(E, D)=\left(\begin{array}{lll}
0 & 0 & 0 \\
0 & 1 & 1 \\
0 & 1 & 1
\end{array}\right), \quad T R L_{3}(E, C)=\left(\begin{array}{lll}
0 & 0 & 1 \\
1 & 0 & 1 \\
1 & 1 & 1
\end{array}\right) \\
T R L_{\text {total }}(E)=T R L_{1}(E, F)+T R L_{2}(E, D)+T R L_{3}(E, C)=\left(\begin{array}{lll}
0 & 0 & 1 \\
1 & 2 & 3 \\
1 & 3 & 3
\end{array}\right)
\end{gathered}
$$

where $T R L_{\text {total }}$ is the total topological relationship. In Eq. $7 a_{22}=2$ indicates that the region $E$ is contact with two regions in its boundary. The topological

Table 1. Topological properties of different regions of the image according to Eq. 5

\begin{tabular}{|c|c|l|}
\hline $\operatorname{TRL}\left(\zeta_{i}, \zeta_{i+1}\right)$ & Primary conditions & Secondary conditions \\
\hline \hline Contact regions & $a_{22}=1, a_{11}=0, a_{21}=0$ & $\begin{array}{l}a_{23}=1, a_{32}=1, a_{33}=1, \\
a_{12}=0, a_{13}=0, a_{31}=0\end{array}$ \\
\hline Inside regions & $a_{21}=1, a_{11}=0, a_{12}=0, a_{22}=0$ & $\begin{array}{l}a_{13}=1, a_{23}=1, a_{31}=1, \\
a_{32}=1, a_{33}=1\end{array}$ \\
\hline Disjoint regions & $a_{11}=0, a_{12}=0, a_{21}=0, a_{22}=0$ & $\begin{array}{l}a_{13}=1, a_{23}=1, a_{31}=1, \\
a_{32}=1, a_{33}=1\end{array}$ \\
\hline
\end{tabular}


Table 2. Topological properties of different regions of the image in Fig. 2

\begin{tabular}{|c|c|c|c|c|}
\hline Region label & Region name & $\begin{array}{c}\text { \#of connected components } \\
\text { (region name) }\end{array}$ & Internal cavity & $\begin{array}{c}\text { Handles / } \\
\text { (region name) }\end{array}$ \\
\hline \hline 1 & $\mathrm{~A}$ & $2 /(\mathrm{bg}),(\mathrm{C})$ & 1 & $1 /(\mathrm{C})$ \\
\hline 2 & $\mathrm{bg}$ & $1 /(\mathrm{A})$ & 1 & $1 /(\mathrm{A})$ \\
\hline 3 & $\mathrm{C}$ & $3 /(\mathrm{A}),(\mathrm{D}),(\mathrm{E})$ & 2 & $2 /(\mathrm{D}),(\mathrm{E})$ \\
\hline 4 & $\mathrm{D}$ & $3 /(\mathrm{C}),(\mathrm{E}),(\mathrm{F})$ & 1 & $1 /(\mathrm{F})$ \\
\hline 5 & $\mathrm{E}$ & $3 /(\mathrm{C}),(\mathrm{D}),(\mathrm{F})$ & 0 & 0 \\
\hline 6 & $\mathrm{~F}$ & $2 /(\mathrm{E}),(\mathrm{E})$ & 0 & 0 \\
\hline
\end{tabular}

similarity $T_{s}$ between each cluster in the topological graph and the corresponding region in the image during evolution is for updating the labels of pixels of each region in the image at each $t$ during evolution process, i.e. it is applied iteratively during curve evolution to update the label of each pixel in an image. $T_{s}$ is determined by subtraction $T R L_{\text {total }}\left(\zeta_{i}(b(x, y))\right)$ from $T R L_{\text {total }}\left(R_{\vec{\gamma}_{i}}(b(x, y))\right)$ during evolution process:

$$
T_{s}=\left\{\begin{array}{l}
0 \text { for } T R L_{\text {total }}\left(\zeta_{i}(b(x, y))\right)=T R L_{\text {total }}\left(R_{\vec{\gamma}_{i}}(b(x, y))\right) \\
1 \quad \text { otherwise }
\end{array}\right.
$$

where $R_{i}$ are the regions inside the curves $\overrightarrow{\gamma_{i}}$ during evolution process. The area and the centroid of each contour are calculated at each $t$ during evolution process and compared with the area and the centroid of corresponding cluster in the topological graph:

$$
A_{i}=\int_{A} d A, \quad C_{x i}=\frac{1}{A} \int_{A} x_{e} d A, \quad C_{y i}=\frac{1}{A} \int_{A} y_{e} d A
$$

where $A_{i}$ are the areas, $C_{x i}$ and $C_{y i}$ are the coordinates of centroid, $x_{e}$ and $y_{e}$ are coordinates of the centroid of the differential element of area $d A$. The prior information is added to the functional energy as topological graph prior term:

$$
E_{g}\left[\left(\overrightarrow{\gamma_{i}}\right)_{i=1}^{N-1}\right]=\underbrace{\alpha\left(\int_{R_{\vec{\gamma}_{i}}}\left(\left|A_{\zeta_{i}}-A_{R_{i}}\right|\right) d x+\left(\left|C_{\zeta_{i}}-C_{R_{i}}\right|\right)+T_{s}\right)}_{\text {Topological graph prior term }}
$$

$E_{g}$ is the energy of the topological graph. $\alpha$ is constant $(\alpha=1$ or 0$)$ to run the algorithm with or without topological graph prior. $A_{\zeta_{i}}, C_{\zeta_{i}}$ are the area and centroid of the clusters in topological graph and $A_{R_{i}}, C_{R_{i}}$ are the area and the centroid of the regions in the image $B$ during the evolution process respectively.

\subsection{Multi-level Set Method}

For image $B$ consists of $N$ regions, each region is represented as $R_{\vec{\gamma}_{i}}(t)=\{b \in$ $\left.R \mid \vec{\gamma}_{i}(b, t)>0\right\}, i=1, \ldots, N$. During curve evolution, for each region $R_{i}$ we assume its complement $R_{i}^{c}\left\{R_{\overrightarrow{\gamma_{1}}}(t), R_{\vec{\gamma}_{1}}(t)^{c} \cap R_{\vec{\gamma}_{2}}, R_{\vec{\gamma}_{1}}(t)^{c} \cap R_{\vec{\gamma}_{2}}^{c} \cap R_{\vec{\gamma}_{3}}(t),\left(\bigcup_{j=1}^{N-1} R_{\vec{\gamma}_{j}}(t)\right)^{c}\right\}$, see [13, 14. The total Euler-Lagrange energy functional can be written as follows: 


$$
E_{\text {total }}\left[\left(\vec{\gamma}_{i}\right)_{i=1}^{N-1}\right]=\int_{R_{\vec{\gamma}_{i}}} \omega_{i}(b) d b+\int_{R_{\vec{\gamma}_{i}^{c}}^{c}} \psi_{i}(b) d b+\lambda \oint_{\overrightarrow{\vec{\gamma}_{i}}} d s+E_{g}
$$

where the first two terms are the data terms of the region $R_{i}$ and $R_{i}^{c}$ which are fully explained in 13, 14, the third term is the regularization term and the fourth term is our proposed prior term. $\omega_{i}$ are the data in $R_{i}$ and $\psi_{i}$ are the data in $R_{i}^{c}$, for more details see [13, [14. $\lambda$ is positive real constant to weight the relative contribution of the energy equation.

\subsection{Curve Evolution}

To minimize Eq11 by curve evolution we compute:

$$
\frac{d \overrightarrow{\gamma_{i}}}{d t}=-\frac{\partial E}{\partial \overrightarrow{\gamma_{i}}}
$$

$\frac{\partial E}{\partial \overrightarrow{\gamma_{i}}}$ are the derivative of functional energy with respect to $\overrightarrow{\gamma_{i}}$ and they are computed as for the standard region computation functional in [15]. Using the result in [15], we get the following evolution equation of the curves $\overrightarrow{\gamma_{i}}$ :

$$
\frac{\overrightarrow{\gamma_{i}}}{\partial t}=-(\omega_{i}(b)-\psi_{i}(b)+\underbrace{\alpha\left[\left(\left|A_{\zeta_{i}}-A_{R_{i}}\right|\right)+\left(\left|C_{\zeta_{i}}-C_{R_{i}}\right|\right)+T_{s}\right]}_{\text {Topological graph prior }}+\lambda k_{i}) \overrightarrow{n_{i}}
$$

where $k_{i}$ are the curvature of zero level set of $\overrightarrow{\gamma_{i}}, \overrightarrow{n_{i}}$ are the external unit normal of the curve, $i \in[1, \ldots, N], j \in[1, \ldots, N]$ and $i \neq j$.

During curve evolution each curve constrains by the topological graph prior information and the curvature term, the topological information are the error of $A$ and $C$, and the state of the topological similarity $T_{s}$. The curvature term is $k_{i} \cdot \vec{n}_{i}$. For $N$-region segmentation we consider $N-1$ evolution curves as mentioned previously. Let $b(x, y)$ be pixels in the image $B(b(x, y) \in B)$ and let $\overrightarrow{\gamma_{i}}(0)$ be an initial curve and $\overrightarrow{\gamma_{i}}(t)$ is a curve in an iteration $t . A \vec{\gamma}_{R_{i}}$ and $C_{\vec{\gamma}_{R_{i}}}$ are updated for each time step during evolution process.

The errors between $A_{\zeta_{i}}$ and $A_{\vec{\gamma}_{R_{i}}}$ and between $C_{\zeta_{i}}$ and $C_{\vec{\gamma}_{R_{i}}}$ should be minimized.

The topological similarity $T_{s}$ defines the label state of the pixel $b(x, y)$ in each region in the image $B$ at time $t+1$ with respect to the label of the same pixel at time $t$. Using Eq. 8, if $T_{s}(b)=0$ at $\vec{\gamma}_{i}(t)$ and $\vec{\gamma}_{i}(t+1)$ then $b \in \vec{\gamma}_{i}$. If $T_{s}(b)=0$ at $\vec{\gamma}_{i}(t)$ and $T_{s}(b)=1$ at $\vec{\gamma}_{i}(t+1)$ then $b \in \vec{\gamma}_{j}, i \neq j, i \in[1, \ldots, N]$ and $j \in[1, \ldots, N]$.

If $b$ is a point of contact between two curves $\left(\overrightarrow{\gamma_{i}}, \vec{\gamma}_{j}\right)$, then the curve will be constrained by the curvature term as follows: If the curvatures are positive $\left(k_{i}(b) \geq 0, k_{j}(b) \geq 0\right)$ this indicates that these curves are retract and not intersect. If $\left(k_{i}(b) \leq 0, k_{j}(b) \geq 0\right)$ this indicates that these two curves will be in the same direction but because $\left|k_{i}(b) \leq k_{j}(b)\right|$, the curve $\vec{\gamma}_{j}$ retracts faster than $\overrightarrow{\gamma_{i}}$ 
and the curves will not intersect. The algorithm of the topological graph prior is describe in Alg. 1. The graph constraint makes the partitioning more precise during evolution process by adding addition constrain information $\left(T_{s}(b)\right.$, area, centroid).

\section{Algorithm 1. Algorithm for computing topological graph prior}

Given an image $B$, consists of $N$ regions.

1. Compute the error of $A$ and $C$ between $\vec{\gamma}_{R_{i}}$ and $\zeta_{i}$ during evolution process:

- During curve evolution at each $t$.

$$
\begin{gathered}
\text { while }\left(\left|A_{\zeta_{i}}-A_{R_{\vec{\gamma}_{i}}}\right|>\epsilon\right) \&\left(\left|C_{\zeta_{i}}-C_{R_{\vec{\gamma}_{i}}}\right|>\epsilon\right) \text { do } \\
\alpha\left[\left(\left|A_{\zeta_{i}}-A_{R_{\vec{\gamma}_{i}}}\right|\right)+\left(\left|C_{\zeta_{i}}-C_{R_{\vec{\gamma}_{i}}}\right|\right)\right] \overrightarrow{n_{i}}
\end{gathered}
$$

end while

2. Compute the similarity of the topological relationship $T_{s}$ :

- During curve evolution (at each $t$ ) for each pixel $b(x, y) \in B$.

- At time $=\mathbf{t}$ :

$$
\forall \mathrm{b}(\mathrm{x}, \mathrm{y}) \in B
$$

if $\overrightarrow{\gamma_{i}}(b)>0$ then

$$
\begin{aligned}
& b(x, y) \in \vec{\gamma}_{i} ; \\
& \text { Find } T R L_{\text {total }}\left(R_{\vec{\gamma}_{i}}(b(x, y))\right) ; \\
& {\left[l_{i}\right]=\left\{b(x, y) \in R_{i} \mid b \sim l\right\} ;}
\end{aligned}
$$

end if

- At time $=\mathbf{t}+\mathbf{1}:$

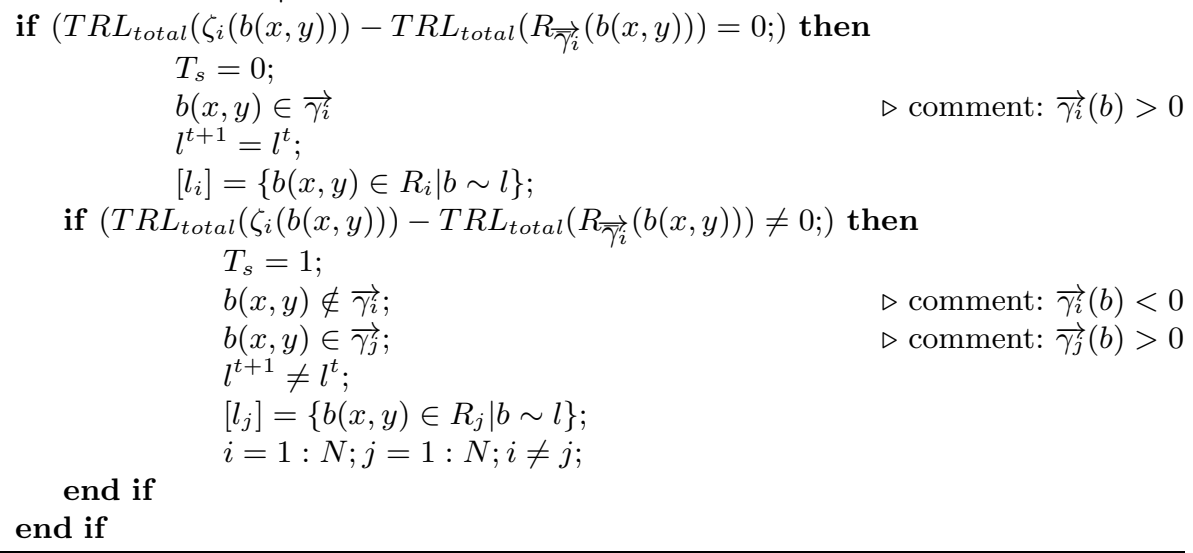

\section{Experiments and Discussion}

In order to explore the advantages and shortcoming of our algorithm with respect to different state-of-the-art multi-region segmentation methods, we conducted 
experiments on diverse medical images. We used in our experiments the MedPix [17, Wesky E Snyder [18, brain web for simulated brain database [19] and other medical images from the internet. All images in the database are 2D MRI images and CT images. The sizes of the images are $150 \times 150$ and $512 \times 512$ for abdominal sections of MRI images and CT images and $181 \times 217$ and $512 \times 512$ for brain sections of MRI images and CT images.

All experiments are conducted in MATLAB using a $2.0 \mathrm{GHz}$ Intel core I3 CPU. Figure 3 shows multi-region segmentation for abdominal and brain MRI images with and without graph prior. The ground truth is obtained by manual segmentation. Figure 3 shows the improvements of our algorithm to capture the overlapped and close gray level regions according to its topological location in the image. The abdominal image in Fig. 3 shows the improvements of our algorithm mainly in the segmentation of aorta, liver and diaphragm. The brain images show the segmentation of the cerebellum, brainstem, white matter and gray matters. In Fig. 3, the segmented regions in the proposed algorithm are labeled by colors according to their topological relationship. The result of the algorithms proposed by 13 and 14 are labeled manually to visualize the differences. Our algorithm resists to noise and gives precise results with less number of iterations and computation time as shown in Table 3. The accuracy of the proposed algorithm depends on the accuracy of the precise extraction of each cluster in the topological graph, i.e. $T_{s}, A$ and $C$ should be computed precisely for each cluster in the topological graph. Our algorithm can be extended to use other features of the clusters to compare them with the regions belong the contours during the evolution process. Dice similarity coefficients (DSC) [20] are computed between the ground truth segmentation and our algorithm as well as the other algorithms to validate the accuracy of our results. Dice coefficient measures the similarity between the manual segmented image and the tested image using:

Table 3. Segmentation accuracy for each database without the effect of noise

\begin{tabular}{|c|c|c|c|}
\hline Algorithm & DSC [17] & DSC [18] & DSC [19] \\
\hline \hline Graph prior (proposed) & $93.56 \%$ & $90.57 \%$ & $94.88 \%$ \\
\hline Without graph prior [13], [14] & $80.64 \%$ & $79.89 \%$ & $82.49 \%$ \\
\hline ChanVese [2] & $61.82 \%$ & $61.6 \%$ & $62.78 \%$ \\
\hline Level set fuzzy based [4] & $40.87 \%$ & $40.1 \%$ & $42.63 \%$ \\
\hline
\end{tabular}

Table 4. Overall segmentation accuracy of all images in database [17, 18], 19] and the average computation time for each frame

\begin{tabular}{|c|c|c|c|}
\hline Algorithm & DSC & \# iteration & Time \\
\hline \hline Graph prior (proposed) & $93 \%$ & 70 & $2.24 \mathrm{~min}$ \\
\hline Without graph prior [13], [14] & $81 \%$ & 70 & $2.87 \mathrm{~min}$ \\
\hline ChanVese [2] & $62 \%$ & 400 & $5.15 \mathrm{~min}$ \\
\hline Level set fuzzy based [4] & $41.2 \%$ & 300 & $3.2 \mathrm{~min}$ \\
\hline
\end{tabular}




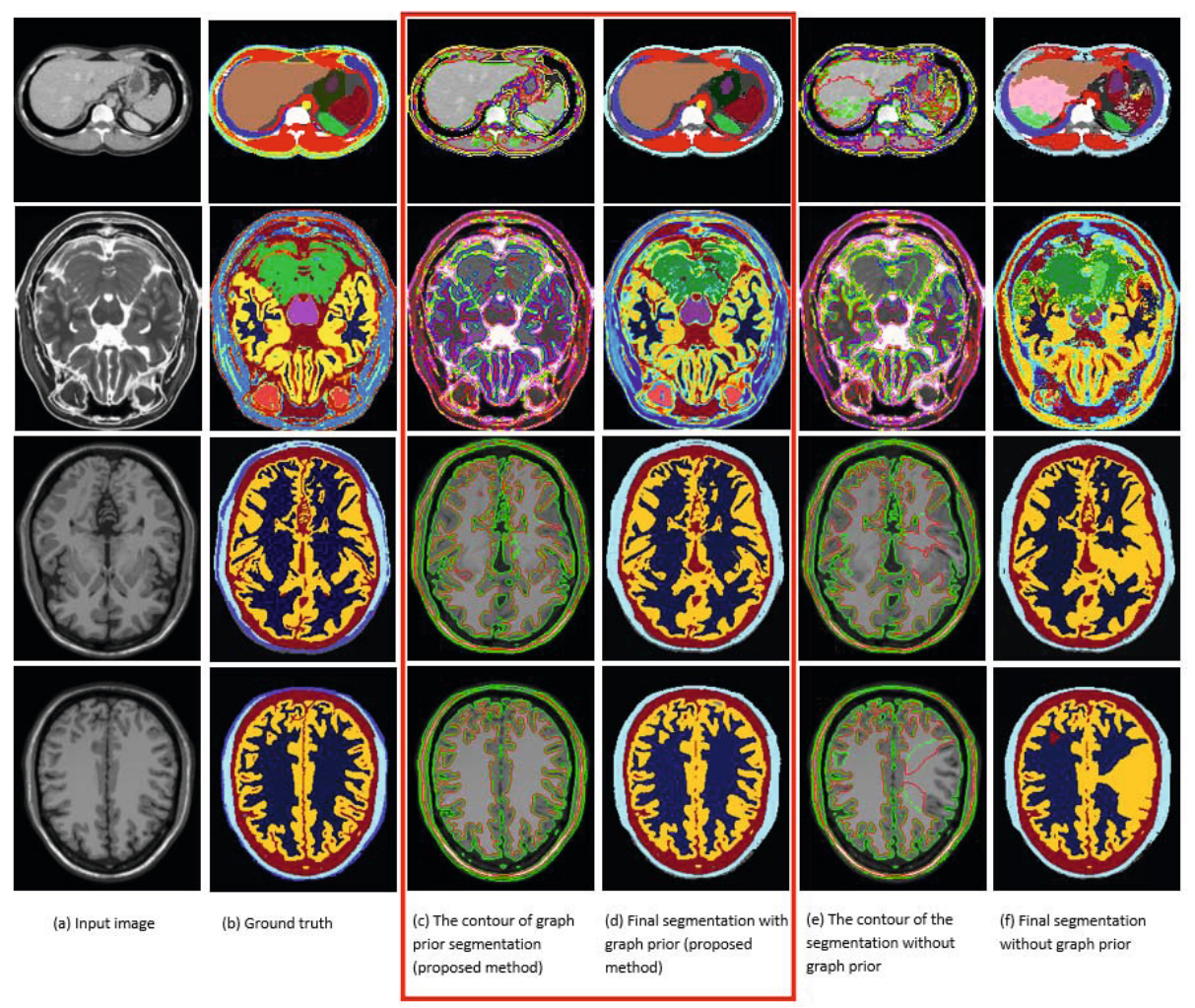

Fig. 3. Multi-region segmentation results. (a) input images (b) ground truth, (c, d) proposed algorithm with graph prior and $(\mathrm{e}, \mathrm{f})$ without graph prior. An example images from database [17, 18], 19.

$$
D S C\left(B_{g t}-B_{t}\right)=\frac{2 P\left(B_{g t}-B_{t}\right)}{P\left(B_{g t}\right)+P\left(B_{t}\right)}
$$

$P\left(B_{g t}-B_{t}\right)$ is the number of overlapping pixels and $P\left(B_{g t}\right)+P\left(B_{t}\right)$ is the sum of the number of pixels in each image. A large DSC indicates higher accuracy. Table 3 shows the accuracy, for six region segmentation, over 130 brain and 43 abdominal images of the database [17, 8 abdominal and 6 brain images of the database [18, and more than 800 brain images of the database [19]. Table 4 shows the overall accuracy of our algorithm and other algorithms without presence of noise. Fig. 4 shows the effect of noise on the overall accuracy using the databases [17, [18], 19] and it can be seen the improvement of our algorithm, compared to other methods.

\subsection{Additional Result}

To verify our approach comparing with the approaches in [13] and [14, we conducted experiments on diverse images that consist two objects with the same 


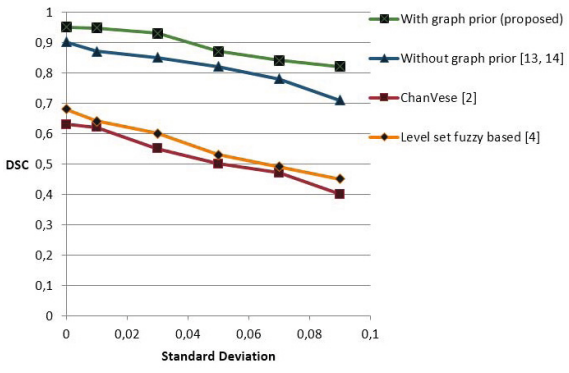

(a) DSC versus noise for 3 regions segmentation

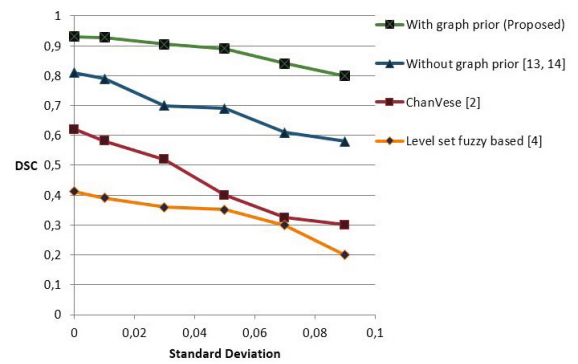

(b) DSC versus noise for 6 regions segmentation

Fig. 4. Effect of gaussian noise on segmentation performance of database [17, 18, 19]

gray level to highlight on the advantages of the proposed approach to segment objects having same gray level whether these are Contiguous objects or not. Figure [5] shows the result of the proposed approach (with topological graph prior) comparing with approaches proposed by [13] and [14] (without topological graph prior) using images consist of two regions from database 21. The first row of Fig. 5 shows the original images and the second row shows the ground truth which are obtained by [21]. The third row shows the final position of the evolving curves for the original images and the fourth row shows the final segmentation of the proposed approach, which are very close to the ground truth. The fifth row shows final position of the evolving curves for the original images and the sixth row shows the final segmentation of [13] and [14]. Our algorithm needs 2 minutes to process the $200 \times 170$ images and the regularization parameter $\lambda$ was set to 0.2 , while the approaches [13] and [14] needs 2.5 minutes to process these images with same regulation parameters. From the result we can see the advantages of our approach to segment the objects regardless to their gray level with less computing time.

\section{Conclusion and Future Work}

We propose to use a topological graph prior in a multi-level set formulation for multi-region segmentation and partitioning. As a high level prior, it gives accurate region partitioning with respect to their topological location and relationship. Several experiments using both medical images and general images demonstrate the advantages of our method. The accuracy of our approach depends on the accuracy of the extraction of the topological graph prior information. As future work, we will focus on improving the extraction of topological graph prior information. The topological graph information will be affected in the presence of strong noise. This limitation can be solved using non-rigid registration of an atlas information to the topological graph. Our approach could be extended using other feature of the topological graph to increase the accuracy of the result. 


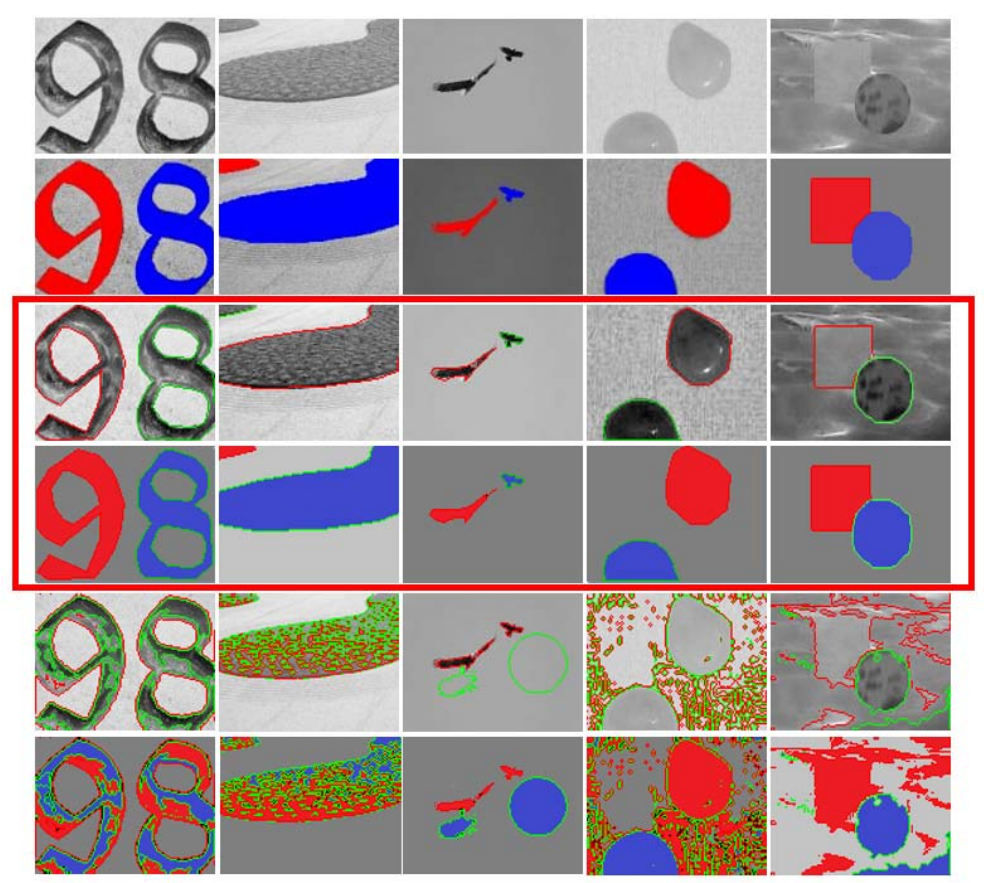

Fig. 5. Multi-region segmentation of multi-objects images, $1^{\text {st }}$ row shows the original images, $2^{\text {nd }}$ row shows the ground truth which are obtained using [21, $3^{\text {rd }}$ and $4^{\text {th }}$ rows show the result of the proposed approach (with the topological graph prior), and $5^{t h}$ and $6^{t h}$ rows show the result of [13] and [14] (without the topological graph prior). An example images from database [21]. The size of the images is $200 \times 170$, after 100 iterations

Acknowledgement. The work was funded by DAAD scholarship (A/10/96106) and MOHESR-Iraq. The authors gratefully acknowledge these supports.

\section{References}

1. Andrews, S., McIntosh, C., Hamarneh, G.: Convex multiregion probabilistic segmentation with shape prior in isometric log-ratio transformation space. In: ICCV, pp. 2096-2103 (2011)

2. Chan, T., Vese, L.: Active contours without edges. IEEE Transaction on Image Processing 10(2), 266-277 (2001)

3. Rathke, F., Schmidt, S., Schnörr, C.: Order Preserving and Shape Prior Constrained Intra-retinal Layer Segmentation in Optical Coherence Tomography. In: Fichtinger, G., Martel, A., Peters, T. (eds.) MICCAI 2011, Part III. LNCS, vol. 6893, pp. 370-377. Springer, Heidelberg (2011)

4. Li, B.N., Chui, C.K., Chang, S., Ong, S.H.: Integrating Spatial Fuzzy Clustering With Level Set Methods For Automated Medical Image Segmentation. ELSEVIER Computer in Biology and Medicine 41, 1-10 (2011) 
5. Suzuki, M., Linguraru, M.G., Summers, R.M., Okada, K.: Analyses of Missing Organs in Abdominal Multi-Organ Segmentation. In: Yoshida, H., Sakas, G., Linguraru, M.G. (eds.) Abdominal Imaging. LNCS, vol. 7029, pp. 256-263. Springer, Heidelberg (2012)

6. Shimizua, A., Ohnoa, R., Ikegamia, T., Kobatakea, H., Nawanob, S., Smutekc, D.: Segmentation of Multiple Organs in Non-Contrast 3D Abdominal CT Images. Int. J. CARS 2, 135-142 (2007)

7. Linguraru, M.G., Pura, J.A., Chowdhury, A.S., Summers, R.M.: Multi-organ segmentation from multi-phase abdominal CT via 4D graphs using enhancement, shape and location optimization. In: Jiang, T., Navab, N., Pluim, J.P.W., Viergever, M.A. (eds.) MICCAI 2010, Part III. LNCS, vol. 6363, pp. 89-96. Springer, Heidelberg (2010)

8. Kohlberger, T., Sofka, M., Zhang, J., Birkbeck, N., Wetzl, J., Kaftan, J., Declerck, J., Zhou, S.K.: Automatic Multi-organ Segmentation Using Learning-Based Segmentation and Level Set Optimization. In: Fichtinger, G., Martel, A., Peters, T. (eds.) MICCAI 2011, Part III. LNCS, vol. 6893, pp. 338-345. Springer, Heidelberg (2011)

9. Okada, T., Linguraru, M.G., Yoshida, Y., Hori, M., Summers, R.M., Chen, Y.-W., Tomiyama, N., Sato, Y.: Abdominal Multi-Organ Segmentation of CT Images Based on Hierarchical Spatial Modeling of Organ Interrelations. In: Yoshida, H., Sakas, G., Linguraru, M.G. (eds.) Abdominal Imaging 2011. LNCS, vol. 7029, pp. 173-180. Springer, Heidelberg (2012)

10. Bazin, P.L., Pham, D.L.: Homeomorphic Brain Image Segmentation with Topological and Statistical Atlases. In: MICCAI 2007 (2007); Medical Image Analysis 12(5), 616-625 (2008)

11. Otsu, N.: A Threshold Selection Method from Gray-Level Histograms. IEEE Transaction on System, Man and Cybernetics 9, 62-66 (1979)

12. Egenhofer, M., Herring, J.: Categorizing Binary Topological Relations between Regions, Lines and Points in Geographic Databases. Technical report, Dept. of Surveying Eng., Univ. of Maine (1991)

13. Mansouri, R., Mitiche, A., Vazquez, C.: Multiregion Competition: A Level Set Extension of Region Competition to Multiple Region Image Partitioning. Computer Vision and Image Understanding 101, 137-150 (2006)

14. Vazquez, C., Mitiche, A., Ayed, I.B.: Image Segmentation as Regularized Clustering: A Fully Global Curve Evaluation Method. ICIP 5, 3467-3470 (2004)

15. Zhu, S.C., Yuille, A.: Region Competition: Unifying Snakes, Region Growing, and Bayes/mdl for Multiband Image segmentation. PAMI 18, 884-900 (1996)

16. Majumdar, A.K., Bhattacharya, I., Saha, A.K.: An Object-Oriented Fuzzy Data Model for Similarity Detection in Image Databases. IEEE Trasaction on Knowledge and Data Engineering 14(5), 1186-1189 (2002)

17. MedPix Medical Image Database (1999)

18. Snyder, W.E.: NC state university Image Analysis Laboratory Database (2002)

19. Cocosco, C.A., Kollokian, V., Kwan, R.K.-S., Evans, A.C.: BrainWeb: Online Interface to a 3D MRI Simulated Brain Database. NeuroImage 5, S425 (1997)

20. Zou, K.H., Warfield, S.K., Bharatha, A., Tempany, C.M.C., Kaus, M.R., Haker, S.J., Wells, W.M., Jolesz, F.A., Kikinis, R.: Statistical Validation of Image Segmentation Quality Based on a Spatial Overlap Index. Academic Radiology 11, 178-189 (2004)

21. Alpert, S., Galun, M., Basri, R., Brandt, A.: Image Segmentation by Probabilistic Bottom-Up Aggregation and Cue Integration. PAMI 34(2), 315-327 (2012) 